\title{
Overview of recent lattice QCD results: phase diagram, fluctuations and strangeness
}

\author{
Jana N. Guenther ${ }^{1, *}$ \\ ${ }^{1}$ Aix-Marseille Université
}

Abstract. In recent years there has been much progress on the investigation of the QCD phase diagram with lattice QCD. This work will focus on the developments in the last few years with physical parameters and low chemical potential which are obtained by extrapolations from zero or imaginary chemical potential.

\section{Introduction}

The investigation of the QCD phase diagram has been a very active topic for many years. Lattice QCD is a very effective, non perturbative tool to solve QCD. Therefore, it is an obvious choice to turn to when instigating the QCD phase diagram. Simulations at finite baryon chemical potential are, however, hindered by the sign problem. Therefore, other methods are necessary to gain insight at finite $\mu_{B}$ physics from lattice QCD. This work will review recent results obtained from lattice QCD simulations with physical parameters at low finite $\mu_{B}$ by extrapolations from zero or imaginary $\mu_{B}$. A more detailed review, including more parameter spaces, can be found in Ref. [1].

Since at $\mu_{B}=0$ the transition is a crossover (Ref. [2-7]), some observables can be discribed by an analytic function in the vicinity of zero. This fact can be exploited, by using results at imaginary or zero chemical potential. A very common technique for extrapolation is the so called Taylor method. The pressure is prametrized as

$$
\frac{p}{T^{4}}=\sum_{j=0}^{\infty} \sum_{k=0}^{\infty} \frac{1}{j ! k !} \chi_{j k}^{B S} \hat{\mu}_{B}^{j} \hat{\mu}_{S}^{k}
$$

with $\hat{\mu}=\frac{\mu}{T}$. The $\chi_{j k}^{B S}$ can be measured on zero $\mu$ lattices. Another expansion from which results will be discussed in this work, is the so called fugacity expansion or sector method. Here the parametrization

$$
\frac{p}{T^{4}}=\sum_{j=0}^{\infty} \sum_{k=0}^{\infty} P_{j k}^{B S} \cosh \left(j \hat{\mu}_{B}-k \hat{\mu}_{S}\right)
$$

is used. Here the expansion coefficients $P_{j k}^{B S}$ have to be determined either from the $\chi_{j k}^{B S}$ or from imaginary chemical potential. In general for simulations with imaginary chemical potential arbitrary fit functions are possible to describe the data at $\mu^{2}<0$. The choice of

\footnotetext{
*e-mail: jana.gunther@univ-amu.fr
} 


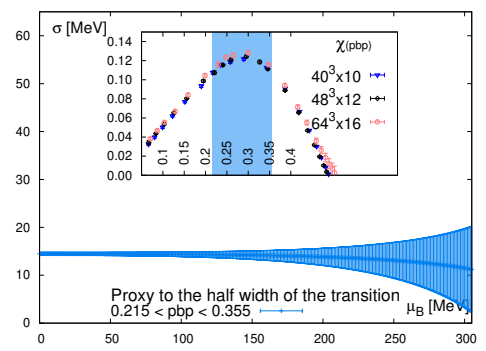

Figure 1. (Ref. [11]) Extrapolation of the half width of the transition to finite baryon chemical potential as defined in equation (3). The insert shows the chiral suceptibility $\chi$ as a function of the chiral condensate $\langle\bar{\psi} \psi\rangle$. The shaded region illustrates $\langle\bar{\psi} \psi\rangle_{c} \pm \Delta\langle\bar{\psi} \psi\rangle / 2$.

fit function is usually guided by the fit quality, sometimes complemented by known physical features. An advantage of the fugacity expansion is its rapid convergence in the hadronic phase and the added information on the particle contend from different sectors. On the other hand the Taylor expansion convergences rapidly in the high temperature limit.

\section{The phase diagram}

The crossover nature of the transition and it temperature has been determined since 2006 with a variety of observables (Ref. [2-9]). More recently the transition temperature has been determined with increased precision in Ref. [10] and Ref. [11]. By now the error on the transition temperature at $\mu_{B}=0$ is much smaller than the width $\sigma$ of the crossover. Therefore it can be determined seperatly. A possible definition, that has been used in Ref. [11] is

$$
\langle\bar{\psi} \psi\rangle\left(T_{c} \pm \sigma / 2\right)=\langle\bar{\psi} \psi\rangle_{c} \pm \Delta\langle\bar{\psi} \psi\rangle / 2
$$

The result, extrapolated to the continuum from three lattice spacings, can be seen in figure 1 up to $\mu_{B}=300 \mathrm{MeV}$.

The behaviour of the transition temperature in the $\mu_{B}$-T-plane can be parameterized by the Taylor expansion as

$$
\frac{T_{c}\left(\mu_{B}\right)}{T_{c}(0)}=1-\kappa_{2}\left(\frac{\mu_{B}}{T_{c}}\right)^{2}-\kappa_{4}\left(\frac{\mu_{B}}{T_{c}}\right)^{4}+O\left(\mu_{B}^{6}\right) .
$$

The odd powers of $\frac{\mu_{B}}{T}$ vanish due to the isospin symmetry. The coefficients $\kappa_{2}$ has been determined in several computations. The more recent ones from Refs. [10-14] are compared in the right side of figure 2 . The green points were obtained from extrapolations with imaginary chemical potential while the blue points were obtained by the Taylor method. Both method yield agreeing results. In addition to $\kappa_{2}$ new results for $\kappa_{4}$ are available from Ref. [10, 11]. While it is clear that $\kappa_{4} \ll \kappa_{2}$ the relative error of $\kappa_{4}$ is more than $100 \%$ and the sign therefore still undetermined.

The extrapolation of the transition temperature to finite $\mu_{B}$ for the choice of $n_{S}=0$ and $n_{Q}=0.4 n_{B}$, to match the condition in heavy ion collisions from Refs. [10, 11] is shown in figure 3. As a comparison several results for the freeze-out temperature (Refs. [15-21] are shown. In a addition, on the right side, a result from Dyson-Schwinger-Equation calculations (Ref. [22]) is shown. The curvature agrees well with the lattice result, while the absolute value is set to previous lattice value, which was determined by a different observable and with a larger error. Therefore the difference does not imply a contradiction between the two calculations. 


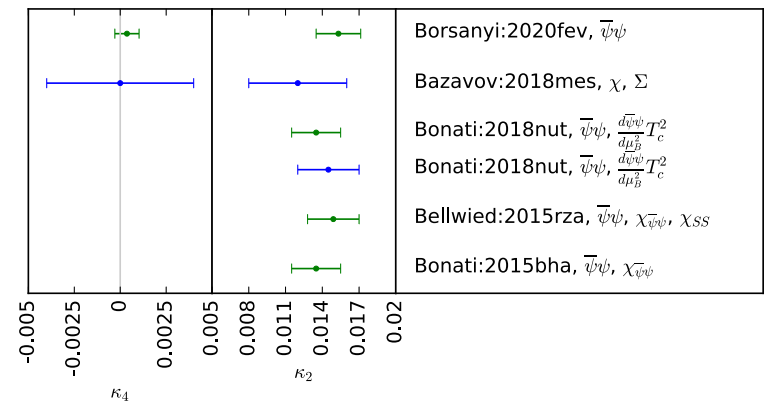

Figure 2. (Ref. [11]) Overview of the different determinations of the $\kappa_{2}$ and $\kappa_{4}$ coefficients as defined in equation (4). The values are taken from Ref. [10-14]. Green points correspond to determinations from imaginary chemical potential, while resuls shown in blue were obtained by the Taylor method.
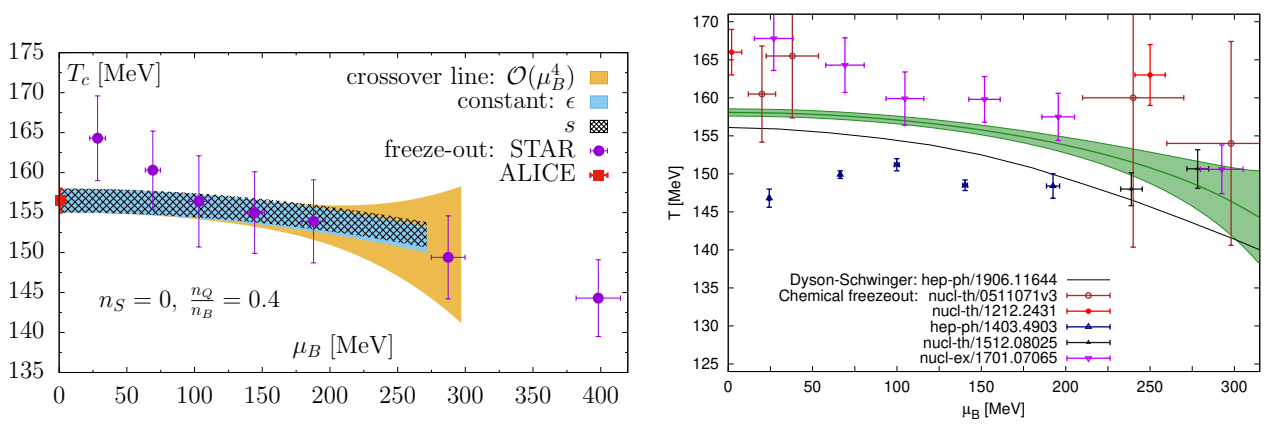

Figure 3. Left: (Ref. [10]) The extrapolation of the transition from the Taylor method compared with the freeze out temperatures from Ref. [20, 21], Right: (Ref. [11]) A comparison of the extrapolated transition temperature from simulations at imaginary chemical potential (green band) and DysonSchwinger-Equations (Ref. [22]). In addition the freeze-out temperatures obtained from heavy ion collisions in Ref. [15-19] are shown.

\section{Fluctuations}

Fluctuations are computed as the derivatives of the pressure with respect to various chemical potentials:

$$
\chi_{i, j, k}^{B, Q, S}=\frac{\partial^{i+j+k}\left(p / T^{4}\right)}{\left(\partial \hat{\mu}_{B}\right)^{i}\left(\partial \hat{\mu}_{Q}\right)^{j}\left(\partial \hat{\mu}_{S}\right)^{k}}, \hat{\mu}_{i}=\frac{\mu}{T}
$$

While fluctuations to various order have previously published for example in Ref. [2327], now new continuum extrapolated results are available in Ref. [28]. The precision of these results is high enough is allow a comparison to different models with detailed studies on inclusion or exclusion of various states for example in a Hadron Resonance Gas model.

The ratios of various fluctuations can be used to express the cumulants of the Baryon number distribution. This offers an observable for comparisons with heavy ion collision measurements of the proton number distribution. At the current precision level this can only be a rough comparison. If the precision is increased in the future, other effects should be taken into account, like the continuum limit on the lattice side, or volume fluctuations and on equilibrium effects on the experimental side.

Figure 4 shows the ratios $R_{31}^{B}\left(T, \mu_{B}\right)=\chi_{3}^{B} / \chi_{1}^{B}=S_{B} \sigma_{B}^{3} / M_{B}, R_{42}^{B}\left(T, \mu_{B}\right)=\chi_{4}^{B} / \chi_{2}^{B}=\kappa_{B} \sigma_{B}^{2}$, $R_{51}^{B}\left(T, \mu_{B}\right)=\chi_{5}^{B} / \chi_{1}^{B}$ and $R_{62}^{B}\left(T, \mu_{B}\right)=\chi_{6}^{B} / \chi_{2}^{B}$ as a function of $R_{12}^{B}\left(T, \mu_{B}\right)=M_{B} / \sigma_{B}^{2}$ evaluated along the transition line. While the two lower order ratios are a continuum estimate from $N_{t}=8$ and $N_{t}=12$ lattices $R_{51}^{B}\left(T, \mu_{B}\right)$ and $R_{62}^{B}\left(T, \mu_{B}\right)$ are computed only on an $N_{t}=8$ lattice. 

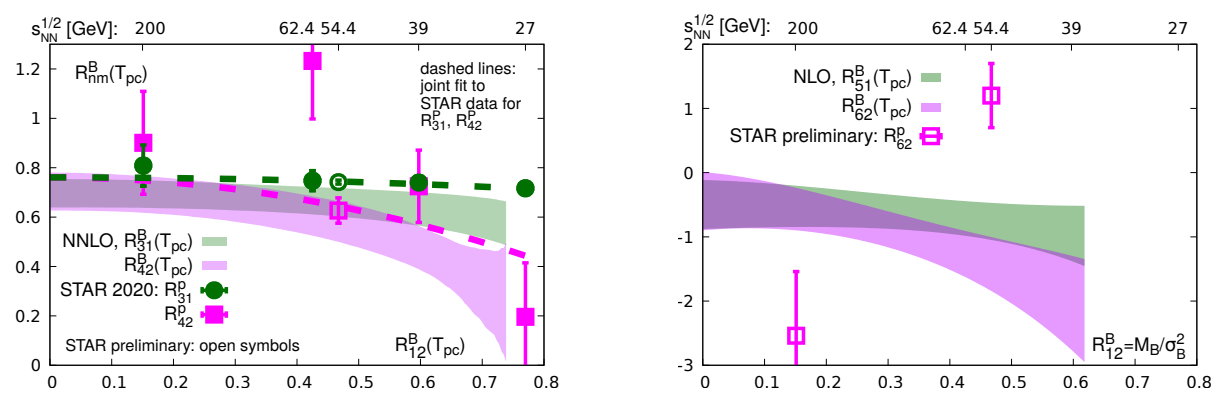

Figure 4. (Ref. [27]) Left: The ratios $R_{31}^{B}\left(T, \mu_{B}\right)=S_{B} \sigma_{B}^{3} / M_{B}$ and $R_{42}^{B}\left(T, \mu_{B}\right)=\kappa_{B} \sigma_{B}^{2}$ as a function of $R_{12}^{B}\left(T, \mu_{B}\right)=M_{B} / \sigma_{B}^{2}$ evaluated along the transition line in comparison to the data from the STAR collaboration (Ref. $[29,30])$. Right: The ratios $R_{51}^{B}\left(T, \mu_{B}\right)$ and $R_{62}^{B}\left(T, \mu_{B}\right)$ as a function of $R_{12}^{B}\left(T, \mu_{B}\right)$ in comparison to the data from the STAR collaboration (Ref. [30]).

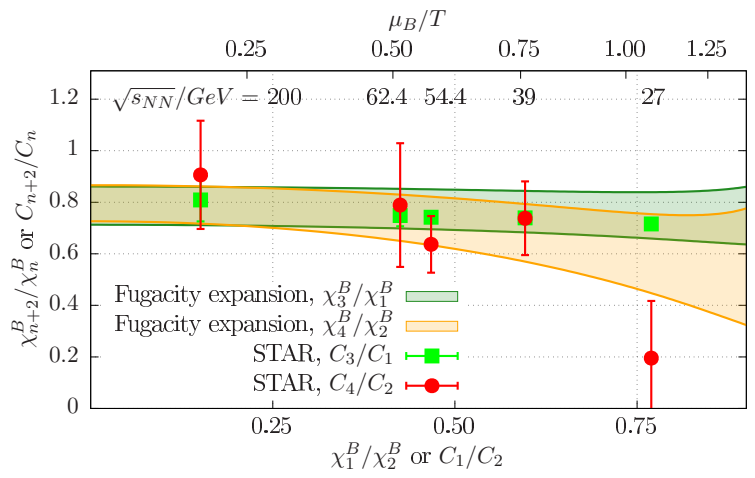

Figure 5. (Ref. [31]) The ratios

$R_{31}^{B}\left(T, \mu_{B}\right)=S_{B} \sigma_{B}^{3} / M_{B}$ and

$R_{42}^{B}\left(T, \mu_{B}\right) \equiv \kappa_{B} \sigma_{B}^{2}$ as a function of $R_{12}^{B}\left(T, \mu_{B}\right)=M_{B} / \sigma_{B}^{2}$ evaluated along the transition line in comparison to the data from the STAR collaboration (Ref. [29]).

A corresponding result from Ref. [31] is shown in figure 5. Here the results are a continuum estimate obtained with a different action on $N_{t}=8,10$ and 12 lattices. The analysis is based on a two dimensional fugacity expansion with imaginary $\mu_{B}$ and $\mu_{S}$ (see equation (2)).

To include strange particles like $K$ or $\Lambda$ in the comparison with lattice calculations, suitable observables have to be constructed. Ref. [32] used the Hadron Resonance Gas (HRG) model to compare different proxies (see left of figure 6). The proxy $\sigma_{\Lambda}^{2} /\left(\sigma_{\Lambda}^{2}+\sigma_{K}^{2}\right.$ ) for the fluctuation ratio $-\frac{\chi_{11}^{B S}}{\chi_{2}^{S}}$ was further investigated both by comparing the HRG and the experimental (see middle of figure 6) results, as well as lattice calculations from both the Taylor and sector method (see right of figure 6).

\section{The equation of state}

Another quantiy that has been investigated for long time is the equation of state. In the following some progress on the baryon number $n_{B}$ will be discussed. When extrapolated to finite $\mu_{B}$ with a Taylor expansion up to $\mu_{B}^{6}$ is shows a surprising behaviour around the transition temperature (see left and middle of figure 7). This behaviour has been observed by different groups and on different data sets (Refs. [25? ]). Ref. [34] could link it to the general convergence of the Taylor series in this region. A possible expansion for the difficulties of the convergence might be that the extrapolation has to cover both the hadronic and the quark gluon plasma phase. Therefore a new extrapolation scheme from imaginary $\mu_{B}$ was proposed that shows a smooth behaviour as can be seen on the right side of figure 7 . 

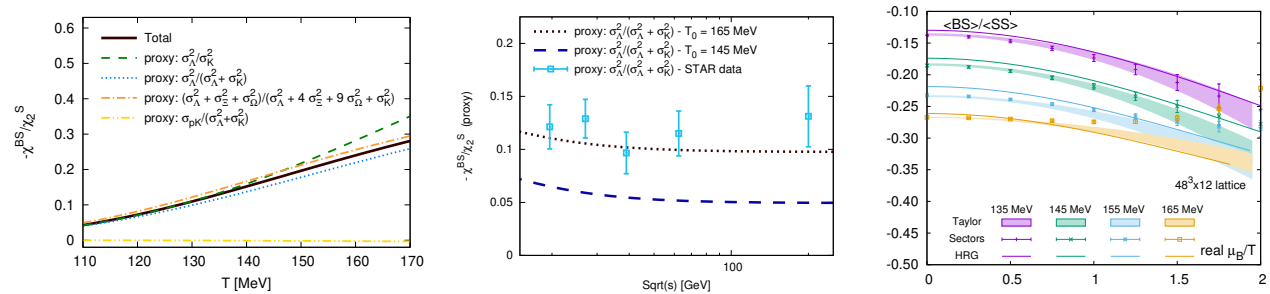

Figure 6. (Ref. [32]) Left: Calculations for different combinations of praticle number cumulants (proxis) that could be measurable in heavy ion collision expermients and the total of $-\frac{\chi_{11}^{B S}}{\chi_{2}^{S}}$ that is accessible in lattice QCD calculations with the Hadron Resonance Gas (HRG) model. Middle: Comparison of the proxy $\sigma_{\Lambda}^{2} /\left(\sigma_{\Lambda}^{2}+\sigma_{K}^{2}\right)$ for two temperatures in the HRG model and experimental results from Ref. [33] Right: Comparison between the Taylor (see equation (1)) and the sector (see equation (2)) method for $-\frac{\chi_{11}^{B S}}{\chi_{2}^{S}}$ on an $48^{3} \times 12$ lattice, as well as results from the HRG model.
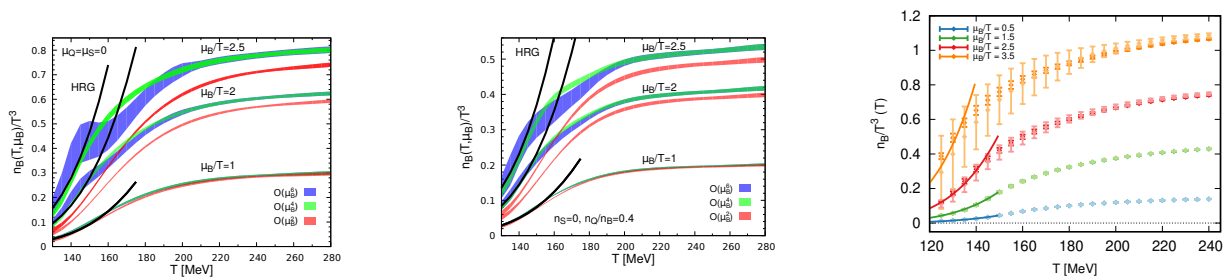

Figure 7. (Ref. [25]) The extrapolation to finite baryon number $n_{B}$ done by the Taylor method (see equation (1)): Left: $\mu_{Q}=\mu_{S}=0$, Middle: $n_{S}=0$ and $n_{Q} / n_{B}=0.4$, Right: (Ref. [34]) The pressure and the baryon number from the resummed extrapolation. The lighter bars show the increased error by the inclusion of a higher order term.

\section{Acknowledgments}

The project leading to this publication has received funding from Excellence Initiative of AixMarseille University - A*MIDEX, a French "Investissements d'Avenir" programme, AMX18-ACE-005.

\section{References}

[1] J.N. Guenther, Eur. Phys. J. A 57, 136 (2021), 2010. 15503

[2] Y. Aoki, G. Endrodi, Z. Fodor, S.D. Katz, K.K. Szabo, Nature 443, 675 (2006), hep-lat/0611014

[3] Y. Aoki, Z. Fodor, S. Katz, K. Szabo, Phys. Lett. B 643, 46 (2006), hep-1at/0609068

[4] Y. Aoki, S. Borsanyi, S. Durr, Z. Fodor, S.D. Katz, S. Krieg, K.K. Szabo, JHEP 06, 088 (2009), 0903.4155

[5] S. Borsanyi, Z. Fodor, C. Hoelbling, S.D. Katz, S. Krieg, C. Ratti, K.K. Szabo (Wuppertal-Budapest), JHEP 09, 073 (2010), 1005 . 3508

[6] T. Bhattacharya et al., Phys. Rev. Lett. 113, 082001 (2014), 1402 . 5175

[7] A. Bazavov et al., Phys. Rev. D85, 054503 (2012), 1111.1710

[8] M. Cheng et al., Phys. Rev. D 74, 054507 (2006), hep-lat/0608013 
[9] A. Bazavov et al., Phys. Rev. D 80, 014504 (2009), 0903.4379

[10] A. Bazavov et al. (HotQCD), Phys. Lett. B795, 15 (2019), 1812.08235

[11] S. Borsanyi, Z. Fodor, J.N. Guenther, R. Kara, S.D. Katz, P. Parotto, A. Pasztor, C. Ratti, K.K. Szabo (2020), 2002.02821

[12] C. Bonati, M. D’Elia, F. Negro, F. Sanfilippo, K. Zambello, Phys. Rev. D 98, 054510 (2018), 1805.02960

[13] R. Bellwied, S. Borsanyi, Z. Fodor, J. Guenther, S.D. Katz, C. Ratti, K.K. Szabo, Phys. Lett. B751, 559 (2015), 1507.07510

[14] C. Bonati, M. D’Elia, M. Mariti, M. Mesiti, F. Negro, F. Sanfilippo, Phys. Rev. D92, 054503 (2015), 1507.03571

[15] A. Andronic, P. Braun-Munzinger, J. Stachel, Nucl. Phys. A772, 167 (2006), nucl-th/0511071

[16] F. Becattini, M. Bleicher, T. Kollegger, T. Schuster, J. Steinheimer, R. Stock, Phys. Rev. Lett. 111, 082302 (2013), 1212.2431

[17] P. Alba, W. Alberico, R. Bellwied, M. Bluhm, V. Mantovani Sarti, M. Nahrgang, C. Ratti, Phys. Lett. B738, 305 (2014), 1403.4903

[18] V. Vovchenko, V.V. Begun, M.I. Gorenstein, Phys. Rev. C93, 064906 (2016), 1512.08025

[19] L. Adamczyk et al. (STAR), Phys. Rev. C96, 044904 (2017), 1701.07065

[20] A. Andronic, P. Braun-Munzinger, K. Redlich, J. Stachel, Nature 561, 321 (2018), 1710.09425

[21] L. Adamczyk et al. (STAR), Phys. Rev. C 96, 044904 (2017), 1701.07065

[22] P. Isserstedt, M. Buballa, C.S. Fischer, P.J. Gunkel, Phys. Rev. D100, 074011 (2019), 1906.11644

[23] C. Schmidt (BNL-Bielefeld), Nucl. Phys. A 904-905, 865c (2013), 1212 . 4278

[24] M. D’Elia, G. Gagliardi, F. Sanfilippo, Phys. Rev. D95, 094503 (2017), 1611.08285

[25] A. Bazavov et al., Phys. Rev. D95, 054504 (2017), 1701.04325

[26] S. Borsanyi, Z. Fodor, J.N. Guenther, S.K. Katz, K.K. Szabo, A. Pasztor, I. Portillo, C. Ratti (2018), 1805 . 04445

[27] A. Bazavov et al., Phys. Rev. D 101, 074502 (2020), 2001.08530

[28] D. Bollweg, J. Goswami, O. Kaczmarek, F. Karsch, S. Mukherjee, P. Petreczky, C. Schmidt, P. Scior (2021), 2107.10011

[29] J. Adam et al. (STAR) (2020), 2001.02852

[30] T. Nonaka (STAR), Measurement of the Sixth-Order Cumulant of Net-Proton Distributions in Au+Au Collisions from the STAR Experiment, in 28th International Conference on Ultrarelativistic Nucleus-Nucleus Collisions (2020), 2002.12505

[31] R. Bellwied, S. Borsanyi, Z. Fodor, J.N. Guenther, S.D. Katz, P. Parotto, A. Pasztor, D. Pesznyak, C. Ratti, K.K. Szabo (2021), 2102.06625

[32] R. Bellwied, S. Borsanyi, Z. Fodor, J.N. Guenther, J. Noronha-Hostler, P. Parotto, A. Pasztor, C. Ratti, J.M. Stafford, Phys. Rev. D 101, 034506 (2020), 1910. 14592

[33] L. Adamczyk et al. (STAR), Phys. Lett. B 785, 551 (2018), 1709.00773

[34] S. Borsányi, Z. Fodor, J.N. Guenther, R. Kara, S.D. Katz, P. Parotto, A. Pásztor, C. Ratti, K.K. Szabó, Phys. Rev. Lett. 126, 232001 (2021), 2102.06660 